\title{
Die kontrollierte Abgabe von Cannabis als wissenschaftliche Forschung aus rechtlicher Sicht
}

Samuel Schweizer *

Das Bundesamt für Gesundheit verweigerte der Universität Bern eine Ausnahmebewilligung für die Abgabe von Cannabis zu Genusszwecken im Rahmen einer wissenschaftlichen Studie. Das Betäubungsmittelgesetz sieht die Abgabe von Betäubungsmitteln ausnahmsweise zu wissenschaftlichen Zwecken vor. Nach der Auffassung des Autors wäre eine begrenzte Studie nach geltendem Recht grundsätzlich bewilligungsfähig.

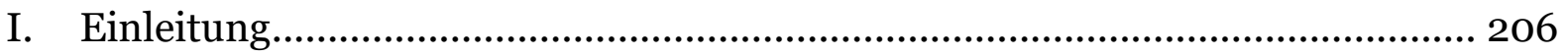

II. Hintergrund und Gegenstand der nicht bewilligten Studie .......................... 206

III. Grundsätzliches Verbot von Cannabis ....................................................... 208

IV. Der Ausnahmetatbestand gemäss Art. 8 Abs. 5 BetmG ................................. 208

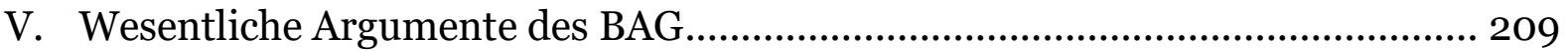

VI. Würdigung des Entscheids des BAG .......................................................211

1. Bedeutung der Notwendigkeit einer ärztlichen Verordnung von Betäubungsmitteln und des medizinischen Zwecks .....................................211

2. Zulässigkeit der nicht-klinischen wissenschaftlichen Forschung nach Art. 8 Abs. 5 BetmG ...................................................................... 212

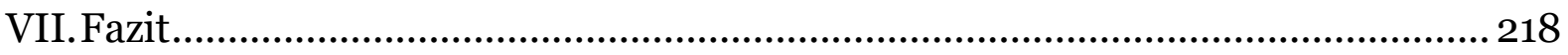

Exkurs: Bewilligung aufgrund eines «Experimentierartikels» de lege ferenda ....... 219

Zitiervorschlag: Samuel Schweizer, Die kontrollierte Abgabe von Cannabis als wissenschaftliche Forschung aus rechtlicher Sicht, in: sui-generis 2018, S. 205

URL: $\quad$ sui-generis.ch/69

DOI: $\quad$ https://doi.org/10.21257/sg.69

* Samuel Schweizer (schweizer[at]poledna.legal), MLaw, ist Rechtsanwalt in Zürich. 


\section{Einleitung}

1 Am 10. Mai 2017 beantragte die Universität Bern beim Bundesamt für Gesundheit (BAG) eine Ausnahmebewilligung für die Abgabe von Cannabis zu Genusszwecken im Rahmen einer wissenschaftlichen Studie. Die Studie sah vor, dass die Teilnehmenden den Cannabis über Apotheken beziehen können. Durch die Studie sollte untersucht werden, wie sich ein regulierter Verkauf von Cannabis auf die Konsumierenden und auf den illegalen Cannabismarkt in der Stadt Bern auswirken würde. ${ }^{1}$

Das BAG lehnte ab und teilte Folgendes mit:

«In der Schweiz sind unter anderem Anbau, Herstellung, Inverkehrbringen, Besitz und Konsum von Cannabis gemäss dem aktuell gültigen Betäubungsmittelgesetz verboten. Für wissenschaftliche Forschungsprojekte oder für die beschränkte medizinische Anwendung können Anbau, Herstellung und Inverkehrbringen von Cannabis ausnahmsweise bewilligt werden. Der Konsum zu Genusszwecken, wie ihn die vorliegende Studie vorsieht und voraussetzt, bleibt aber in jedem Fall verboten und kann nach Betäubungsmittelgesetz nicht bewilligt werden, auch nicht im Rahmen von wissenschaftlichen Studien. Aus diesem Grund konnte das BAG die Ausnahmebewilligung nicht erteilen. (...)

Um solche Studien bewilligen zu können, müsste das Betäubungsmit-

BAG, Medienmitteilung vom 14. November 2017, Studie der Universität Bern zum Cannabiskonsum nicht bewilligungsfähig. telgesetz mit einem Experimentierartikel ergänzt werden. Damit könnte das Verbot des Konsums von Cannabis zu Genusszwecken während einer bestimmten Zeit und beschränkt auf bestimmte Orte und einen bestimmten Adressatenkreis für wissenschaftlich begleitete Studien ausser Kraft gesetzt werden.»²

3 Das BAG ergänzte, dass ein solcher «Experimentierartikel» ein gesundheitspolitisches Anliegen sei.3 Die Universität Bern akzeptierte den Entscheid des BAG und legte keine Rechtsmittel ein. 4 Der vorliegende Beitrag soll untersuchen, ob die rechtliche Analyse des BAG korrekt ist oder ob eine derartige Studie bereits auf der Grundlage des geltenden Rechts hätte bewilligt werden können.

\section{Hintergrund und Gegenstand der nicht bewilligten Studie}

4 Cannabis ist das in der Schweiz am häufigsten konsumierte verbotene Betäubungsmittel.5 In den letzten Jahren haben u.a. Uruguay (2013) sowie die USBundesstaaten Colorado und Washington (beide 2012), Oregon und Alaska (beide 2014), sowie Kalifornien, Nevada und Massachusetts (alle 2016) Cannabis entkriminalisiert. Weitere (Bundes-) Staaten planen die Entkriminalisierung. Eine Entkriminalisierung des Cannabiskonsums wird in der Schweiz seit Jahren diskutiert. Uruguay war der erste

\footnotetext{
BAG, Medienmitteilung (Fn. 1).

BAG, Medienmitteilung (Fn. 1).

Uni Bern akzeptiert ein Nein des Bundes bei der Cannabis-Studie, Derbund.ch vom 12. Dezember 2017.

5 Siehe Über die Studie, Hintergrund (www.scriptstudie.ch).
} 
Staat, der die Substanz Ende 2013 legalisierte. 6

diesem Hintergrund hätte die Studie vom Clinical Trials Unit (CTU) Bern, einer Abteilung der medizinischen Fakultät der Universität Bern, durchgeführt werden sollen. ${ }^{7}$ Methodisch hätte die Studie auf zwei zufällig (randomisiert) ausgewählten Vergleichsgruppen von regelmässigen Cannabiskonsumenten basiert, die sich in dem Punkt unterschieden hätten, dass die eine Gruppe früher als die andere Zugang zu reguliertem Cannabis erhielt. Den einzelnen Mitgliedern der Vergleichsgruppen wären regelmässig verschiedene quantitative und qualitative Fragen gestellt worden. Die Befragungen wären anonym gewesen. ${ }^{8}$

6 Die Website, die für die Studie aufgeschaltet wurde, fasste das Studiendesign wie folgt zusammen:

«In einer ersten Phase wird eine randomisiert-kontrollierte Studie durchgeführt. Sie hat das Ziel, die gesundheitlichen und sozialen Auswirkungen des Cannabiskonsums in zwei verschiedenen Gruppen zu vergleichen. Eine Gruppe kann von Beginn weg Cannabis in Apotheken kaufen, die andere Gruppe kann dies in den ersten 6 Monaten noch nicht. Im Anschluss an die randomisierte Studie erhalten alle Studienteilnehmenden die Möglichkeit, für mindestens 12 und maximal 30 Monate Cannabis in der Apotheke zu erwerben. Diese

Siehe Über die Studie, Hintergrund (www.scriptstudie.ch).

7 Siehe Über die Studie, Hintergrund (www.scriptstudie.ch).

8 Siehe Über die Studie, Studiendesign (www.scriptstudie.ch). zweite Phase erlaubt uns, die sich hieraus ergebenden gesellschaftlichen Auswirkungen auf die Stadt vor und nach dem bewilligten Verkauf zu vergleichen.»9

7 Die Berner Studie wollte, so steht es im zweiten Satz, die gesundheitlichen und sozialen Auswirkungen von zwei Vergleichsgruppen untersuchen (nämlich von regelmässigen Cannabiskonsumenten, welche Cannabis quasi-legal erwerben, und solchen, die Cannabis illegal erwerben). Im letzten Satz ist die Rede davon, dass die gesellschaftlichen Auswirkungen auf die Stadt untersucht werden sollen.

8 Die folgenden Outcome-Parameter hätten vor, während und nach der Einführung der regulierten Cannabisabgabe erfasst werden sollen:

- Veränderungen im Konsumverhalten wie Konsumkompetenz oderproblematischer Konsum, Risikowahrnehmung und Befinden.

- Wissen über Cannabis, seine Wirkungen und Gefahren des problematischen Konsums.

- Spitaleinweisungen, Bussen und Verzeigungen, Entwicklungen auf dem Schwarzmarkt.

- Ökonomische Aspekte, die im Zusammenhang mit Cannabiskonsum stehen. ${ }^{10}$
$9 \overline{\text { Siehe Über die Studie, Studiendesign (www.script- }}$ studie.ch).

10 Universität Bern, Studie zum Regulierten Cannabis Verkauf in Stadt Berner Apotheken, Kurzfassung Februar 2017, S. 2. 
9 Es zeigt sich insgesamt, dass nicht gesundheitliche, sondern gesellschaftliche Auswirkungen des Cannabis-Konsums im Vordergrund der Studie hätten stehen sollen.

\section{Grundsätzliches Verbot von Cannabis}

10 Gemäss Art. 2 lit. a BetmG11 gilt Cannabis, «sowie Stoffe und Präparate, die auf [dessen] Grundlage hergestellt werden oder eine ähnliche Wirkung wie diese[s] haben», als Betäubungsmittel.

11 Gemäss Art. 8 Abs. 1 lit. d BetmG dürfen Betäubungsmittel des Wirkungstyps Cannabis «weder angebaut, eingeführt, hergestellt noch in Verkehr gebracht werden». Cannabis ist mit anderen Worten verboten (so auch die Sachüberschrift zu Art. 8 BetmG: «Verbotene Betäubungsmittel»).

In Anhang 1 BetmVV-EDI'2, dem gesetzlichen Verzeichnis der Betäubungsmittel, psychotropen Stoffe, usw. wird sodann Cannabis ab einem THC-Gehalt von 1 Prozent, Cannabisharz (Haschisch), Cannabissamen und Cannabisstecklinge mit einem THC-Gehalt von mindestens 1 Prozent aufgeführt.

13 Der unbefugte Anbau, Handel, Besitz, usw. wird gemäss Art. 19 Abs. 1 BetmG mit Freiheitsstrafe bis zu drei Jahren oder Geldstrafe geahndet. Der gewerbsmässige Handel mit Betäubungsmitteln

11 Bundesgesetz über die Betäubungsmittel und die psychotropen Stoffe vom 3. Oktober 1951 (SR 812.121).

12 Verordnung des EDI über die Verzeichnisse der Betäubungsmittel, psychotropen Stoffe, Vorläuferstoffe und Hilfschemikalien vom 30. Mai 2011 (SR 812.121.11). wird mit mindestens einem Jahr Freiheitsstrafe bestraft (Abs. 2). Wer unbefugt Betäubungsmittel vorsätzlich konsumiert oder wer zum eigenen Konsum eine Widerhandlung im Sinne von Art. 19 BetmG begeht, wird dagegen nur mit Busse bestraft (Art. 19a Abs. 1 BetmG). Vorbereitungshandlungen für den eigenen Konsum geringfügiger Mengen sind nicht strafbar (Art. 19b Abs. 1 BetmG), wobei 10 Gramm Cannabis als geringfügige Menge gelten (Abs. 2). Der Konsum von Cannabis bleibt aber strafbar, da auch der Konsum von Cannabis in einem vereinfachten Verfahren mit einer Ordnungsbusse von CHF 100 bestraft wird (Art. 28b Abs. 1 und 2 BetmG).

14 Zusammengefasst kann festgehalten werden: Anbau, Handel, Besitz und Konsum von Cannabis sind nach dem BetmG grundsätzlich verboten. Jedoch hat der Gesetzgeber für Cannabis im Vergleich zu andern Betäubungsmitteln Sondervorschriften vorgesehen, die klar zum Ausdruck bringen, dass Cannabis im Vergleich zu anderen grundsätzlich ebenfalls verbotenen Betäubungsmitteln als weniger gravierend betrachtet wird.

\section{Der Ausnahmetatbestand gemäss Art. 8 Abs. 5 BetmG}

15 Vom grundsätzlichen Verbot von Cannabis in Art. 8 Abs. 1 BetmG sieht derselbe Artikel in Abs. 5 eine Ausnahme vor. Gemäss dieser Vorschrift kann das BAG Ausnahmebewilligungen für den Anbau, die Einfuhr, die Herstellung und das Inverkehrbringen erteilen, wenn kein internationales Abkommen entgegensteht und diese Betäubungsmittel der wissenschaftlichen Forschung, der Arzneimit- 
telentwicklung oder der beschränkten medizinischen Anwendung dienen.

Eine Ausnahmebewilligung liegt vor, wenn von der im Normalfall geltenden Regelung - insbesondere von einer bestimmten polizeilichen Vorschrift - in einzelnen Sonderfällen gestützt auf eine gesetzliche Ermächtigung abgewichen werden darf. 13 dieser Bestimmung um eine KannVorschrift handelt. Selbst wenn die Voraussetzungen der Bestimmung erfüllt sind, ist das BAG nicht verpflichtet, eine Ausnahmebewilligung $\mathrm{zu}$ erteilen. ${ }^{14}$ Es handelt sich um einen Fall von Entschliessungsermessen, da das Gesetz den Eintritt der Rechtsfolge beim Vorliegen bestimmter Voraussetzungen nicht zwingend vorschreibt. ${ }^{15}$ Auch wenn eine Vorschrift der zuständigen Behörde einen Ermessensspielraum einräumt, bedeutet dies aber nicht, dass sie in ihrer Entscheidung völlig frei wäre. Sie ist vielmehr an die Verfassung gebunden und muss insbesondere das Rechtsgleichheitsgebot, das Verhältnismässigkeitsprinzip und die Pflicht zur Wahrung der öffentlichen Interessen befolgen. Ausserdem sind Sinn und Zweck der gesetzlichen Ordnung auch bei Ermessensentscheiden zu beachten. ${ }^{16}$ Das bedeutet mit anderen Worten, dass das grundsätzliche Cannabisverbot gemäss Art. 8 Abs. 1 BetmG nicht durch jede wissenschaftli-

13 Ulrich Häfelin/Georg Müller/Felix Uhlmann, Allgemeines Verwaltungsrecht, 7. Aufl., Zürich/ St. Gallen 2016, Rz. 2665.

14 Gustav Hug-Beeli, Kommentar zum Bundesgesetz über die Betäubungsmittel und die psychotropen Stoffe vom 3. Oktober 1951, Basel 2015 (zit. als BetmG-Komm), Art. 8 N 54.

15 Häfelin/Müller/Uhlmann (Fn. 13), Rz. 398.

16 Häfelin/Müller/Uhlmann (Fn. 13), Rz. 409. che Forschung ausgehebelt werden darf, sondern der verfolgte legitime Zweck (wie z.B. die wissenschaftliche Forschung) von einiger Bedeutung sein und im öffentlichen Interesse liegen muss. ${ }^{17}$ Letztlich ist also eine Interessenabwägung notwendig.

18 Im Übrigen sieht Art. 8 Abs. 5 BetmG nur zwei kumulative Bedingungen vor für die Ausnahmebewilligung für den Anbau, die Einfuhr, die Herstellung und das Inverkehrbringen von grundsätzlich verbotenen Betäubungsmitteln:

1. Die Betäubungsmittel «dienen» der wissenschaftlichen Forschung, der Arzneimittelentwicklung oder der beschränkten medizinischen Anwendung (Zweckbindung).

2. Es darf kein internationales Abkommen der Ausnahmebewilligung entgegenstehen.

\section{Wesentliche Argumente des BAG}

19 In der Verfügung vom 13. November 2017, welche dem Autor vorliegt, begründete das BAG die Ablehnung des Gesuchs für eine Ausnahmebewilligung nach Art. 8 Abs. 5 BetmG im Wesentlichen wie folgt:

1. Der Zweck des Vorhabens besteht in der wissenschaftlichen Forschung im Sinne von Art. 8 Abs. 5 BetmG. 18

2. Es stehen keine internationalen Abkommen in grundsätzlicher Weise entgegen. ${ }^{19}$ Dieser Auffassung schliesst sich der Autor an. Es soll

17 Hug-Beeli, BetmG-Komm (Fn. 14), Art. 8 N 67.

18 Verfügung vom 13. November 2017, E. 22.

19 Verfügung vom 13. November 2017, E. 23. 
deshalb auf diese Voraussetzung nicht mehr weiter eingegangen werden.

3. Die Erteilung der Bewilligung scheiterte jedoch daran, dass nach Ansicht des BAG auch im Rahmen einer Ausnahmebewilligung gemäss Art. 8 Abs. 5 BetmG nicht «generell» von den übrigen Vorgaben des Betäubungsmittelrechts abgewichen werden könne.

4. Gegenstand der Ausnahmebewilligung gemäss Art. 8 Abs. 5 BetmG sei die Aufhebung des Verbots des Anbaus, der Einfuhr, der Herstellung oder des Inverkehrbringens verbotener Betäubungsmittel. ${ }^{20}$ Dagegen biete diese Bestimmung keinen Raum für «Pilotversuche» im Rahmen einer «experimentellen Gesetzgebung», bei der die Grundordnung während einer bestimmten Zeit ausser Kraft gesetzt werde, um evidenzbasierte Entscheidgrundlagen für spätere, definitiv gedachte Erlasse zu schaffen. ${ }^{21}$ Solche ausdrückliche Experimentierartikel gäbe es z.B. in Art. 68quater IVG $^{22}$, aber eben nicht im BetmG. Zwar könne das BAG in Abweichung von Art. 8 Abs. 1 BetmG das Inverkehrbringen von Cannabis zum Zweck der wissenschaftlichen Forschung ausnahmsweise erlauben. Davon unberührt bleibe jedoch «die Anwendbarkeit aller weiteren auf das konkrete Vorhaben anwendbaren Vorschriften des Betäubungsmittel-

20 Verfügung vom 13. November 2017, E. 24.

21 Verfügung vom 13. November 2017, E. 25.

22 Bundesgesetz über die Invalidenversicherung vom 19. Juni 1959 (SR 831.20). gesetzes und des Ausführungsrechts».23

5. Gemäss Art. 13 BetmG dürfe die Abgabe von Betäubungsmitteln in Apotheken nur aufgrund einer ärztlichen Verordnung erfolgen.24 Art. 11 BetmG halte jedoch fest, dass Ärzte verpflichtet sind, Betäubungsmittel nur in dem Umfang zu verwenden, abzugeben und zu verordnen, wie dies nach den anerkannten Regeln der medizinischen Wissenschaften notwendig sei. ${ }^{25}$ Mit Blick auf die Lehre sei die Verwendung aus medizinischer Sicht allerdings nur dann notwendig, «wenn damit Krankheiten gelindert oder Schmerzen bekämpft werden sollen.» An einer medizinischen Indikation fehle es insbesondere, wenn ein Betäubungsmittel zu reinen Genusszwecken oder zur Erholung verwendet werde, ${ }^{26}$ wobei zu beachten sei, dass der Gesetzgeber die Legalisierung des Cannabiskonsums wiederholt abgelehnt hat. ${ }^{27}$

6. Selbst wenn man von diesen grundlegenden Hürden absähe, liegt aufgrund der Tatsache, dass eine unbegrenzte Anzahl Personen in die Studie eingeschlossen werden könne und folglich auch die benötigte Menge Cannabis unbestimmt sei, kein Sonder-, sondern ein Regelfall vor. Der Regelfall aber ist das Verbot von Cannabis. ${ }^{28}$ Eine Erteilung einer Ausnahmebewilligung hätte jedenfalls voraussetzt, dass die Anzahl der

23 Verfügung vom 13. November 2017, E. 25.

24 Verfügung vom 13. November 2017, E. 29.

25 Verfügung vom 13. November 2017, E. 26.

26 Verfügung vom 13. November 2017, E. 26.

27 Verfügung vom 13. November 2017, E. 27.

28 Verfügung vom 13 . November 2017, E. $32 \mathrm{ff}$. 
teilnehmenden Personen sowie die benötigte Menge Cannabis von Beginn weg festgelegt und begrenzt gewesen wäre. ${ }^{29}$

7. Schliesslich werde für die beschränkte medizinische Anwendung gemäss Art. 28 Abs. 2 lit. d BetmSV3o eine schriftliche Erklärung der Patientin oder des Patienten vorausgesetzt, wonach sie oder er mit der Anwendung einverstanden ist. Dies gelte zwar nach dem Gesetzeswortlaut nicht für die wissenschaftliche Forschung. Da ein ähnliches Erfordernis auch für die betäubungsmittel- und die heroingestütze Behandlung von abhängigen Personen gelte (Art. 9 und 21 BetmSV), sei auch im Kontext der wissenschaftlichen Forschung notwendig, dass die Identität der Teilnehmenden der Bewilligungsbehörde bekannt gegeben werde. ${ }^{1} \mathrm{Zu}$ dem sei im gewählten Studiendesign nicht sichergestellt, dass keine unbefugte Weitergabe von Cannabis namentlich an Minderjährige - erfolge, da bis zu 24 Gramm Cannabis pro Monat für Konsum in Privaträumen abgegeben werde. ${ }^{2}$ Damit werde die Zielsetzung des BetmG einer lückenlosen Kontrolle der Betäubungsmittel vom Anbau bis zum Konsum nicht genügend Rechnung getragen. 33

Zusammenfassend hielt das BAG fest, dass das BetmG das Inverkehrbringen

\footnotetext{
29 Verfügung vom 13. November 2017, E. 34.

3о Verordnung über Betäubungsmittelsucht und andere suchtbedingte Störungen vom 25. Mai 2011 (SR 812.121.6).

31 Verfügung vom 13. November 2017, E. $36 \mathrm{f}$.

32 Verfügung vom 13. November 2017, E. 38.

33 Verfügung vom 13. November 2017, E. 38.
}

von Cannabis zu nichtmedizinischen Zwecken (d.h. zum Genusskonsum) auch nicht im Rahmen der wissenschaftlichen Forschung erlaube. 34 Zwar bestehen neben diesen grundlegenden Hindernissen auch Probleme bezüglich persönlicher und mengenmässiger Beschränkung der Studie, fehlende Identifizierung der Teilnehmenden und fehlender Schutz gegen Weitergabe des Cannabis an Dritte. Das BAG war jedoch der Meinung, dass diese Vorbehalte (wohl) mit einer Anpassung des Studiendesigns hätten ausgeräumt werden können. 35

\section{Würdigung des Entscheids des BAG}

21 Das BAG begründet die Ablehnung im Wesentlichen damit, dass ein Forschungsprojekt, bei welchem Cannabis zu Genusszwecken abgegeben werde, unter dem geltenden BetmG nicht bewilligungsfähig ist. Dabei stützt es sich insbesondere auf Art. 11 i.V.m. 13 BetmG, wonach die Abgabe in Apotheken nur zulässig sei, sofern ein Arzt ein Betäubungsmittel aufgrund einer medizinischen Indikation verschrieben habe. Das bedeutet, dass letztlich nur klinische Forschung im Hinblick auf eine medizinische Nutzung mit verbotenen Betäubungsmitteln aufgrund von Art. 8 Abs. 5 BetmG bewilligt werden könnte.

\section{Bedeutung der Notwendigkeit einer ärztlichen Verordnung von Betäubungsmitteln und des medizinischen Zwecks}

22 Sofern sich die Argumentation des BAG auf Art. 11 und 13 BetmG stützt, wonach Ärzte und Apotheken Betäubungsmittel nur verordnen und abgeben dürfen, so-

34 Verfügung vom 13. November 2017, E. 45.

35 Verfügung vom 13. November 2017, E. 46. 
weit dies nach den anerkannten Regeln der medizinischen Wissenschaften notwendig ist, ist jedoch auch Art. 14 Abs. 2 BetmG zu beachten. Diese Vorschrift sieht vor, dass Institute, die der wissenschaftlichen Forschung dienen, mit Bewilligung des Kantons «nach Massgabe des Eigenbedarfs Betäubungsmittel» anbauen, beziehen, lagern und verwenden dürfen. Dieser Vorschrift ist keine Beschränkung auf medizinische Zwecke zu entnehmen. Der Umfang der bewilligten Handlungen ist dort vielmehr auf den Forschungszweck beschränkt. In diesem Sinne muss die Beschränkung auf den «Eigenbedarf» verstanden werden. Würde dieser Ausdruck die Abgabe von Betäubungsmitteln an Dritte grundsätzlich beschränken, wäre auch klinische Forschung mit Patienten unmöglich.

Im Rahmen von Art. 11 und 13 BetmG stellt sich deshalb nur die Frage, ob diese Artikel es verbieten, Betäubungsmittel in Apotheken abzugeben. Wäre dies der Fall, müsste die Abgabe durch das Forschungsinstitut selber (bzw. seine Hilfspersonen) auf der Grundlage von Art. 14 BetmG erfolgen.

Die grundlegende Frage ist nicht, ob und gegebenenfalls unter welchen Umständen die zur Durchführung der Studie vorgesehenen Medizinalpersonen Cannabis abgeben dürfen, sondern ob wissenschaftliche Forschung im Sinne von Art. 8 Abs. 5 BetmG bewilligungsfähig ist, bei welcher Cannabis zu nichtmedizinischen Zwecken verwendet wird (nicht-klinische Forschung mit Cannabis). Dabei ist insbesondere die Frage zu prüfen, ob Forschung zulässig ist, bei der Cannabis an Studienteilnehmer zu Genusszwecken abgegeben wird.
2. Zulässigkeit der nicht-klinischen wissenschaftlichen Forschung nach Art. 8 Abs. 5 BetmG

25 Wie soeben dargelegt ist die Frage zu beantworten, ob der Begriff der wissenschaftlichen Forschung im Sinne von Art. 8 Abs. 5 BetmG auch nicht-klinische Forschung einschliesst, bei welcher Cannabis wie in der Berner Studie zu Genusszwecken an Studienteilnehmende abgegeben wird.

\section{a) Grammatikalische Auslegung}

26 Der Begriff der wissenschaftlichen Forschung setzt sich aus zwei Wörtern zusammen: Wissenschaft ist gemäss Duden eine «Wissen hervorbringende forschende Tätigkeit in einem bestimmten Bereich»,36 Forschung ist dagegen «das Arbeiten an wissenschaftlichen Erkenntnissen».37 Zusammengefasst geht es also um die Generierung von Wissen. Gemäss Hug-Beeli wird unter wissenschaftlicher Forschung allgemein verstanden, was nach Inhalt und Form als ernsthafter planmässiger Versuch zur Ermittlung der Wahrheit anzusehen ist. ${ }^{8}$ Einen einheitlichen Wissenschaftsbegriff gibt es nicht. 39 Der Begriff darf insbesondere vom Staat nicht zu eng ausgelegt werden, denn dieser hat die Freiheit der wissenschaftlichen Forschung zu beachten (Art. 20 BV). ${ }^{40}$

27 Der Wortlaut alleine legt damit meines Erachtens eher eine weite Auslegung des Begriffs der wissenschaftlichen Forschung nahe. Eine Beschränkung auf klinische Forschung lässt sich dem Begriff nicht entnehmen.

36 Duden.de, Wissenschaft.

37 Duden.de, Forschung.

38 Hug-Beeli, BetmG-Komm (Fn. 14), Art. 8 N 67.

39 Hug-Beeli, BetmG-Komm (Fn. 14), Art. 8 N 67.

40 Hug-Beeli, BetmG-Komm (Fn. 14), Art. 8 N 67. 

auslegung ist auch dem Grundsatz der Einheit der Rechtsordnung und eines möglichst widerspruchsfreien Zusammenwirkens der verschiedenen Rechtsgebiete Rechnung zu tragen. ${ }^{41}$ Deshalb kann auch berücksichtigt werden, wie der Begriff der wissenschaftlichen Forschung in anderen Gesetzen und Rechtsgebieten verstanden wird.

29 Der Begriff der wissenschaftlichen Forschung erscheint prominent in Art. 2 lit. a FIFG42. Er wird in dieser Vorschrift als «die methodengeleitete Suche nach neuen Erkenntnissen» definiert.

Interessant ist in diesem Kontext, dass das BetmG selber mit Art. 3j einen Artikel über die Forschungsförderung enthält. In diesem wird ausdrücklich auf das Forschungsgesetz Bezug genommen. Gemäss dieser Bestimmung kann der Bund im Zusammenhang mit Betäubungsmitteln Forschungsvorhaben betreffend Wirkungsweise abhängigkeitserzeugender Stoffe, Ursachen und Auswirkungen suchtbedingter Störungen, präventiven und therapeutischen Massnahmen, Verhinderung oder Verminderung suchtbedingter Störungen oder Wirksamkeit von Wiedereingliederungsmassnahmen fördern. Aus dieser Aufzählung wird jedenfalls klar, dass Forschung im Sinne von Art. 3j BetmG nicht nur medizinischer oder naturwissenschaftlicher Art sein kann, sondern - wenn es um Ursachen und Auswirkungen geht - auch sozialwissenschaftlicher Art.

41 BGE 143 II 8 E. 7.3.

42 Bundesgesetz über die Förderung der Forschung und der Innovation vom 14. Dezember 2012 (SR 420.1).
31 Auch das Humanforschungsgesetz $(\mathrm{HFG})^{43}$ kennt eine ähnliche Definition wie das FIFG. Forschung wird in Art. 3 lit. a definiert als «methodengeleitete $\mathrm{Su}$ che nach verallgemeinerbaren Erkenntnissen». In der Botschaft zu diesem Gesetz präzisierte der Bundesrat den Begriff der Forschung wie folgt:

«Der Begriff Forschung ist definiert als die methodengeleitete Suche nach verallgemeinerbaren Erkenntnissen. Die Präzisierung 'methodengeleitet' verweist auf die Anwendung von wissenschaftlich anerkannten Vorgehensweisen zur Gewinnung der gesuchten Erkenntnisse. Dabei kann es sich sowohl um natur- als auch um sozialwissenschaftliche Methoden handeln. Die zu gewinnenden Erkenntnisse müssen zudem verallgemeinerbar sein, d.h. sie müssen auch über den Kontext des Forschungsprojekts hinaus Gültigkeit besitzen und dürfen nicht einen nur individuellen Bezug aufweisen. Die Verallgemeinerbarkeit wird z.B. mittels einer genügend hohen Fallzahl sowie einer realitätsnahen Forschungsanlage angestrebt. Nicht unter die Suche nach verallgemeinerbaren Erkenntnissen ist ein Wissenszuwachs ausschliesslich bei einer Einzelperson (z.B. im Rahmen der Aus- und Weiterbildung) zu subsumieren.» 44

32 Das HFG basiert auf einem Verfassungsartikel über Forschung am Menschen (Art. $118 b$ BV). In der Botschaft dazu äusserte sich der Bundesrat zum Begriff der Forschung wie folgt:

43 Bundesgesetz über die Forschung am Menschen vom 30. September 2011 (SR 810.30).

44 Botschaft vom 21. Oktober 2009 zum Bundesgesetz über die Forschung am Menschen (BBl 2009 8045), 8092. 
«Im vorliegenden Entwurf wird ,Forschung' umschrieben als Tätigkeit, mit der neue, verallgemeinerbare Erkenntnisse gewonnen werden sollen. Die Suche nach neuen Erkenntnissen muss systematisch, methodengeleitet und überprüfbar sein, um als (wissenschaftliche) Forschung gelten zu können. Forschung bezieht sich somit auf Erkenntnisgewinn, der wissenschaftlichen Anforderungen entspricht bzw. zumindest beansprucht, das zu tun. $\mathrm{Ob}$ die Art des Erkenntnisgewinns den wissenschaftlichen Anforderungen genügt oder nicht, richtet sich grundsätzlich nach den Standards der jeweiligen Forschergemeinschaft (Scientific Community).

Für die Festlegung der Grenze zwischen Forschung und Praxis gibt es verschiedene Ansätze. Der vorliegende Entwurf knüpft an das Ziel an, das mit der betreffenden Tätigkeit verfolgt wird. Wird mit dieser (wissenschaftliche) Erkenntnis angestrebt, so handelt es sich um Forschung. Steht ein anderes Ziel (z.B. Hilfe für eine Patientin oder einen Patienten) im Zentrum, so liegt grundsätzlich keine Forschung vor.» 45

Zusammengefasst lässt sich zum Begriff der (wissenschaftlichen) Forschung Folgendes festhalten:

1. Ziel der wissenschaftlichen Forschung ist die Gewinnung neuer Erkenntnisse bzw. neuen Wissens.

2. Dabei werden die Methoden einer jeweiligen Forschergemeinschaft

45 Botschaft vom 12. September 2007 zum Verfassungsartikel über die Forschung am Menschen (BBl 2007 6713), 6720.
(Scientific Community) beachtet, wodurch die Erkenntnisse überprüfbar werden.

3. Die Erkenntnisse, die gewonnen werden sollen, müssen verallgemeinerbar sein.

4. Es können natur- oder sozialwissenschaftliche Methoden angewandt werden (so explizit das HFG).

34 Nach diesen Massstäben ergibt sich für die Berner Studie das Folgende:

- Überwiegend sozialwissenschaftlicher Charakter der Studie: Die Studie hätte im Wesentlichen die Auswirkungen auf Gesundheit, Konsumverhalten sowie mit dem Konsum verbundene ökonomische Aspekte der regulierten Cannabisabgabe erfasst. Während die menschliche Gesundheit - wenn man so will - der Sphäre der naturwissenschaftlichen Forschung zugeschlagen werden kann, handelt es sich bei Konsumverhalten und ökonomischen Folgen der regulierten Abgabe um gesellschaftliche Auswirkungen. Diese beiden Aspekte werden mit sozialwissenschaftlicher Methodik erfasst.

- Methodik: Die Studie hätte ein Setting mit zwei zufällig (randomisiert) ausgewählten Gruppen umfasst, wobei die eine Gruppe erst sechs Monate später Zugang zu (legalem) Cannabis erhalten hätte. Allen Teilnehmern wären regelmässig qualitative und quantitative Fragen gestellt worden. Die Befragung hätte anonym stattgefunden. Ein solches Setting entspricht meines Erachtens grundsätzlich grundlegenden, heute gängigen me- 
thodischen Standards. Das Setting mit zwei Kontrollgruppen, dem Einbezug von quantitativen Fragestellungen und die Anonymisierung erlauben die Verallgemeinerung der Erkenntnisse.

Zusammengefasst handelt es sich meines Erachtens bei der Studie um eine sozialwissenschaftliche Fragestellung. Die Methodik entspricht grundsätzlich der in dieser Wissenschaftsgattung üblichen Vorgehensweise; entsprechend sollten die Erkenntnisse verallgemeinerbar sein.

36 Damit kann als Zwischenfazit festgehalten werden, dass die grammatische Auslegung des Begriffs der wissenschaftlichen Forschung nicht der Annahme entgegensteht, dass auch die Forschung der Berner Studie darunter subsumiert werden könnte. In der Folge ist zu überprüfen, ob sich aus einer historischen, systematischen und teleologischen Auslegung Anhaltspunkte ergeben, die eine andere Schlussfolgerung nahelegen.

\section{b) Historische Auslegung}

37 Art. 8 Abs. 5 BetmG wurde mit der Gesetzesrevision vom 20. März 2008 neu gefasst. Er ist seit 1. Juli 2011 in Kraft. Ziel bei der Revision war in erster Linie, dass der Anwendungsfall der Arzneimittelentwicklung und der beschränkten medizinischen Anwendung für alle Betäubungsmittel geöffnet wird (in einer früheren Version bezog sich die beschränkte medizinische Anwendung auf gewisse Betäubungsmittel; Cannabis gehörte nicht dazu).

38 Der ursprüngliche Entwurf der zuständigen Kommission sah folgenden Wortlaut vor:
Das Bundesamt für Gesundheit kann Ausnahmebewilligungen für die Verwendung der Betäubungsmittel nach den Absätzen 1 und 3 in der wissenschaftlichen Forschung oder für eine beschränkte medizinische Anwendung erteilen, wenn:

a. die Voraussetzungen zur Guten Herstellungspraxis, zur Abgabe nicht zugelassener Arzneimittel, zur Guten Laborpraxis oder zur Guten Praxis der klinischen Versuche erfüllt sind;

b. die ethischen Grundsätze und Empfehlungen berücksichtigt sind; und

c. diese Verwendungen im Einklang mit den internationalen Übereinkommen stehen. 46

39 Dieser Vorschlag wurde allerdings nicht ins Gesetz aufgenommen. Die schliesslich von den Räten verabschiedete Fassung verzichtete insbesondere darauf, dass die Voraussetzungen zur Guten Herstellungspraxis, zur Abgabe nicht zugelassener Arzneimittel, zur Guten Laborpraxis und zur Guten Praxis der klinischen Versuche erfüllt werden müssen. Die schliesslich angenommene Fassung wurde von der Verwaltung im Auftrag der Kommission ausgearbeitet. Der Kommission ging es darum, «therapeutische Optionen nicht durch eine unsorgfältige Formulierung oder durch ein explizites Verbot im Betäubungsmittelgesetz strikt zu verunmöglichen oder eine Anwendung dieser Stoffe mit einem unzumutbaren administrativen Aufwand zu belasten».47 Im Vordergrund stand also wiederum die klinische Ver-

$46 \overline{\text { Entwurf Bundesgesetz über die Betäubungsmittel }}$ und psychotropen Stoffe (BBl 2006 8629).

47 AB 2008 N 74, NR Gilli. 
wendung, doch machte sich die Kommission allgemein Sorgen, dass sinnvolle künftige «Anwendungen» durch unzumutbaren administrativen Aufwand verunmöglicht werden könnten.

Selbst wenn der ursprüngliche Vorschlag Gesetz geworden wäre, wäre für die Berner Studie eine Bewilligung nicht per se ausgeschlossen gewesen: Zwar hätten entweder die Voraussetzungen zur Guten Herstellungspraxis, zur Abgabe nicht zugelassener Arzneimittel, zur Guten Laborpraxis oder zur Guten Praxis der klinischen Versuche eingehalten werden müssen. Es ist aber nicht ersichtlich, wieso zumindest die Voraussetzungen zur Guten Herstellungspraxis nicht prinzipiell im Fall der Studie hätten eingehalten werden können. 48

41 Die Hauptstossrichtung der Revision war, wie bereits erwähnt, nicht die Anwendungen für die wissenschaftliche Forschung, sondern beschränkte medizinische Anwendungen und die Arzneimittelentwicklung. Entsprechend äusserte sich der Bericht der zuständigen Ratskommission wie folgt:

«Nach Absatz 5 wird neu die Möglichkeit für Ausnahmebewilligungen für die beschränkte medizinische Anwendung für alle verbotenen Stoffe, einschliesslich Hanf, grundsätzlich zugelassen. Dies sollte vor allem Multiple Sklerose-, Krebs- und Aidspatienten und -patientinnen zugutekommen.»49

48 Man beachte, dass die Einhaltung der verschiedenen Guten Praxen nicht kumulativ erforderlich gewesen wäre, sondern alternativ («oder») bzw. soweit einschlägig.

49 Parlamentarische Initiative Teilrevision des Betäubungsmittelgesetzes, Bericht der Kommission
42 Damit wurde aber keine Aussage über die wissenschaftliche Forschung getätigt, sondern über die beschränkte medizinische Anwendung. Insbesondere lässt sich nicht daraus schliessen, dass der Begriff der wissenschaftlichen Forschung eine Einengung erfahren hätte. Der legitime Zweck der wissenschaftlichen Forschung war nämlich bereits in der Fassung gemäss Bundesgesetz vom 20. März 1975 (in Kraft seit 1. August 1975) und in der Urfassung von 1951 enthalten.50 Zur Tragweite des Vorbehalts für die wissenschaftliche Forschung äusserte sich indessen keine der ursprünglichen Botschaften ${ }^{51}$. Gemäss Huber soll der historische Gesetzgeber damit Forschung im Laboratorium gemeint haben; er bezieht sich dabei auf Botschaften des Bundesrats aus den Jahren 1927 und 1951.52 Der Autor konnte aber in keiner der Botschaften, die Huber in seinem Aufsatz zitierte, einen Beleg für diese Behauptung finden. Ohnehin rückt ein derartiger Wille des historischen Gesetzgebers in den Hintergrund, nachdem inzwischen Jahrzehnte vergangen sind.

43 Jedenfalls vertrat Albrecht vor der letzten Revision von Art. 8 Abs. 5 BetmG die Auffassung, dass sich weder aus dem Gesetzeswortlaut noch aus der Entstehungsgeschichte ergebe, dass mit dem Begriff der

für soziale Sicherheit und Gesundheit des Nationalrates (BBl 2006 8573), 8608.

50 BetmG, Stand am 26. Oktober 2004.

51 Botschaft vom 12. April 1951 über die Revision des Bundesgesetzes (BBl 1951 I 829), 855, und Botschaft vom 9. Mai 1973 betreffend die Änderung des Bundesgesetzes über die Betäubungsmittel (1973 I 1348), 1363.

$5^{2}$ Christian Huber, Die gesetzliche Grundlage einer kontrollierten Heroinabgabe, SJZ 88/1992 S. 48, FN 3. 
wissenschaftlichen Forschung bloss Laborversuche gemeint sein sollen.53

\section{c) Systematische Auslegung}

44 Die Ausnahmebestimmung des Art. 8 Abs. 5 BetmG sieht neben dem Zweck der wissenschaftlichen Forschung, für welchen eine Ausnahme bewilligt werden kann, alternativ auch die Zwecke der Arzneimittelentwicklung und der beschränkten medizinischen Anwendung vor.

45 Wenn der Begriff der wissenschaftlichen Forschung, wie es der Auffassung des BAG entspricht, so ausgelegt wird, dass nur klinische Forschung zu medizinischen Zwecken darunter subsumiert werden kann, so hätte dieser Begriff neben der Arzneimittelentwicklung und der beschränkten medizinischen Anwendung meines Erachtens gar keine eigenständige Bedeutung mehr.

Das Gesetz darf aber im Zweifelsfall nicht so ausgelegt werden, dass eine Variante des Ausnahmetatbestands im Ergebnis redundant ist. Es ist nicht davon auszugehen, dass der Gesetzgeber überflüssige Tatbestandsvarianten geschaffen hat.

\section{d) Teleologische Auslegung}

47 Gemäss dessen Art. 1 soll das BetMG:

- Dem unbefugten Konsum von Betäubungsmitteln und psychotropen Stoffen vorbeugen, namentlich durch Förderung der Abstinenz; Spannungsfeld zwischen Hilfe und Repression, in: BAG (Hrsg.), Ärztliche Verschreibung von Betäubungsmitteln, Bern 1996, S. 58 ff., 62.
- die Verfügbarkeit von Betäubungsmitteln und psychotropen Stoffen zu medizinischen und wissenschaftlichen Zwecken regeln;

- Personen vor den negativen gesundheitlichen und sozialen Folgen suchtbedingter Störungen der Psyche und des Verhaltens schützen;

- die öffentliche Ordnung und Sicherheit vor den Gefahren schützen, die von Betäubungsmitteln und psychotropen Stoffen ausgehen;

- kriminelle Handlungen bekämpfen, die in engem Zusammenhang mit Betäubungsmitteln und psychotropen Stoffen stehen.

48 Die Zwecke des Gesetzes sind entsprechend vielfältig. Das geltende BetmG ist kein reines Repressionsgesetz, wenn auch die Abstinenz nach wie vor ein wichtiges Ziel ist. 54 Wenn das Gesetz aber «vor den negativen gesundheitlichen und sozialen Folgen suchtbedingter Störungen der Psyche und des Verhaltens schützen» will, dann verfolgt es auch sozialpolitische Ziele. Es geht hier darum, die Allgemeinheit vor den sozialschädlichen Auswirkungen von suchtbedingten Störungen zu schützen, womit wiederum eruiert werden können muss, welche Substanzen überhaupt zu suchtbedingten Störungen führen bzw. mithin sozialschädliche Auswirkungen haben können.

49 Die Berner Studie hätte hier ansetzen und die sozialen und gesundheitlichen Auswirkungen einer kontrollierten Cannabisabgabe untersuchen sollen. Dies

54 Thomas Fingerhuth/Stephan Schlegel/Oliver Jucker, BetmG Kommentar, 3. Aufl., Zürich 2016, Art. $1 \mathrm{~N} 5$. 
steht zwar in einem gewissen Widerspruch zum Ziel der Förderung der Abstinenz von Betäubungsmitteln. Andererseits dient es aber dem in Art. 1 BetmG gleichwertig genannten Gesetzeszweck des Schutzes vor negativen sozialen und gesundheitlichen Begleiterscheinungen des Konsums. Zudem gefährdet der mit dem Cannabisverbot einhergehende Schwarzmarkt die öffentliche Ordnung und Sicherheit. Insofern wird mit der Untersuchung der Auswirkungen einer kontrollierten Abgabe auch untersucht, welchen Beitrag eine Entkriminalisierung zur Verbesserung der öffentlichen Ordnung und Sicherheit leisten könnte.

Der Zweck des Gesetzes und des Ausnahmetatbestands wäre jedoch dann nicht mehr eingehalten, wenn durch die Studie die Ausnahme quasi zur Regel würde.55 Das Bundesamt für Justiz (BJ) äusserte sich in einem Rechtsgutachten zur kontrollierten Abgabe harter Drogen an Schwerstsüchtige zu dieser Thematik. ${ }^{6}$ Es folgerte, dass aufgrund der Ausnahmebestimmung des BetmG (des Vorläufers des heutigen Art. 8 Abs. 5 BetmG) eine Abgabe von grundsätzlich verbotenen Betäubungsmitteln nur für «ein zeitlich beschränktes Versuchsprogramm mit einer kleinen Anzahl von Abhängigen» erlaubt werden könnte.57 Nach der Beendigung eines solchen Programms müssten, so das BJ, die Ergebnisse evaluiert werden, woraufhin eine Revision des Gesetzes im Hinblick auf eine Drogenab-

55 Häfelin/Müller/Uhlmann (Fn. 13), Rz. 2674.

${ }_{56}$ Bundesamt für Justiz, Kontrollierte Abgabe harter Drogen. Rechtsgutachten im Auftrag des BAG, in: BAG (Hrsg.), Ärztliche Verschreibung von Betäubungsmitteln, Bern 1996, S. 64 ff.

57 Bundesamt für Justiz, Rechtsgutachten (Fn. 56), S. 76. gabe in grösseren Umfang ins Auge gefasst werden könnte. $5^{8}$

51 Wenn das BJ in diesem Rechtsgutachten auch grundsätzlich skeptisch gegenüber der Heroinabgabe an Schwerstsüchtige blieb, so ist dem Gutachten auch klarerweise zu entnehmen, dass eine in zeitlicher und persönlicher Hinsicht klar beschränkte, versuchsweise Abgabe unter Art. 8 Abs. 5 BetmG subsumiert werden könnte. Dabei kann die Versuchsanordnung auch den Zweck haben, in kleinem und kontrolliertem Rahmen zu erproben, ob eine Gesetzesrevision angezeigt ist. Die damals vom BJ diskutierte Heroinabgabe war überdies prinzipiell nicht zeitlich befristet. Dies ist ein gewichtiger Unterschied zur Studie der Universität Bern. Deshalb spricht meines Erachtens vieles dafür, dass die zeitlich und persönlich beschränkte Cannabisabgabe einer teleologischen Auslegung des BetmG nicht widerspricht.

\section{Fazit}

52 Die Auslegung von Art. 8 Abs. 5 BetmG liefert keine Hinweise, dass ein sozialwissenschaftliches Setting nicht grundsätzlich im Sinne dieser Bestimmung als «wissenschaftliche Forschung» verstanden werden kann.

53 Solange das Forschungsvorhaben in sachlicher Hinsicht auf Cannabis beschränkt gewesen und darüber hinaus auch in zeitlicher, persönlicher und räumlicher Hinsicht eng begrenzt geblieben wäre, ist die nicht-klinische Forschung nach Art. 8 Abs. 5 BetmG grundsätzlich bewilligungsfähig.

${ }_{58} \overline{\text { Bundesamt für Justiz, Rechtsgutachten (Fn. 56), }}$ S. 76. 
54 Nach dem Gesagten hätte eine nichtklinische Studie, bei der Cannabis an die Studienteilnehmenden zu Genusszwecken abgegeben wird, um die sozialen und gesundheitlichen Auswirkungen einer kontrollierten Abgabe zu untersuchen, grundsätzlich bewilligt werden können. Wie sich aus den Erwägung des BAG ergibt, stellten sich aber zusätzliche Fragen:

1. Hätten die Studienteilnehmenden vorab definiert und gemeldet werden müssen?

2. Wie hätte die gesamthaft abgegebene Menge vorab definiert werden können?

3. Mit welchen zusätzlichen Massnahmen hätte verhindert werden können, dass das abgegebene Cannabis an unbefugte Dritte weitergereicht wird?

4. Ist die Abgabe in einer öffentlichen Apotheke ohne ärztliches Rezept möglich? Oder hätte die Abgabe unmittelbar durch das Institut erfolgen müssen?

Nach Auffassung des Autors - und wohl auch des BAG59 - stellt jedoch keine dieser Fragen ein unüberwindbares Hindernis dar, das nicht durch eine Anpassung des Studiendesigns hätte beseitigt werden können.

Die Begründung des BAG, dass die Bewilligung rechtlich aus grundsätzlichen Überlegungen nicht möglich gewesen sei, ist deshalb nicht korrekt. Wenn die entscheidende Behörde ein Ermessen nicht ausübt, das ihr das Gesetz einräumt, liegt

59 Verfügung vom 13. November 2017, E. 46 a.E. eine Ermessensunterschreitung vor. ${ }^{60}$ Die Ermessensunterschreitung stellt eine Rechtsverletzung dar. ${ }^{61}$ Das BAG hätte, wo ihm ein Ermessen zukommt, sachliche Unterscheidungen treffen und den besonderen Umständen des konkreten Falles angemessene Rechtsfolgen anordnen müssen. ${ }^{62}$ Stattdessen wurde die Bewilligungsfähigkeit für derartige wissenschaftliche Forschung in prinzipieller Art und Weise verneint.

\section{Exkurs: Bewilligung aufgrund eines «Experimentierartikels» de lege ferenda}

57 Infolge der ablehnenden Verfügung des BAG beschloss die Kommission für soziale Sicherheit und Gesundheit des Nationalrates, «eine Kommissionsinitiative zu ergreifen, um das BetmG dahingehend zu ergänzen, dass auch wissenschaftliche Projekte/Versuche durchgeführt werden können, die dem Zweck dienen, innovative Regulierungsansätze zum gesellschaftlichen Umgang mit dem Freizeitkonsum von Cannabis zu erproben». ${ }^{63}$ Eine Mehrheit der Kommission des Ständerats stimmte einer Motion zu, welche den Bundesrat «beauftragt zu prüfen, wie im Rahmen der geltenden Gesetzgebung befristete wissenschaftliche Studien zur Erprobung innovativer Regulierungsansätze zum gesellschaftlichen Umgang mit dem Konsum von Cannabis bewilligt werden können».64

60 Häfelin/Müller/Uhlmann (Fn. 13), Rz. 439.

61 Häfelin/Müller/Uhlmann (Fn. 13), Rz. 439.

62 Häfelin/Müller/Uhlmann (Fn. 13), Rz. 440.

63 Parlamentarische Initiative, Experimentierartikel als Grundlage für Studien zur regulierten Cannabis-Abgabe (18.402).

64 Motion, Experimentierartikel als Grundlage für Studien zur regulierten Cannabis-Abgabe (17.4210); Medienmitteilung der SGK-NR vom 18. Mai 2018, Für Cannabis-Studien, aber gegen Legalisierung. 
58 In seiner Verfügung verwies das BAG als Beispiel für einen Experimentierartikel auf Art. 68quater IVG.65 Dieser lautet wie folgt:

«Das Bundesamt kann zum Zweck der Eingliederung befristete Pilotversuche bewilligen, die von den Bestimmungen dieses Gesetzes abweichen können. Es hört vorgängig die Eidgenössische Kommission für die Alters-, Hinterlassenen- und Invalidenversicherung an.»

59 Daran könnte sich der Experimentierartikel im BetmG orientieren. Er kann meines Erachtens relativ allgemein gehalten werden und könnte entsprechend in etwa wie folgt lauten:

«Das Bundesamt kann im Rahmen von örtlich, zeitlich und persönlich beschränkten Pilotversuchen die Abgabe verbotener Betäubungsmittel des Wirkungstyps Cannabis bewilligen, um die gesellschaftlichen und gesundheitlichen Auswirkungen des Genusskonsums dieser Betäubungsmittel zu erforschen. Solche Pilotversuche müssen wissenschaftlich begleitet sein.»

60 Die Einzelheiten solcher Versuche wären durch den Bundesrat in allgemeiner Weise in einer Verordnung und im Anwendungsfall durch das Bundesamt zu regeln.

65 Vorstehend, V. 